\title{
Estenosis congénita de venas pulmonares y malformación vascular broncopulmonar
}

\author{
Congenital pulmonary vein stenosis and bronchopulmonary vascular malformation \\ Jesús De Rubens-Figueroa ${ }^{*}$, José J. Bobadilla-Chávez² y Sara A. Solórzano-Morales ${ }^{3}$ \\ ${ }^{1}$ Laboratorio de Ecocardiografía; ${ }^{2}$ Servicio de Cardiología; ${ }^{3}$ Servicio de Radiología. Instituto Nacional de Pediatría, Ciudad de México, México
}

\begin{abstract}
Resumen
El objetivo es mostrar el diagnóstico y la evolución de una paciente con estenosis de venas pulmonares y secuestro pulmonar. Se trata de una niña de 1 año de edad, con bronconeumonías de repetición, acrocianosis, 2R intenso, cardiomegalia, hipertensión venocapilar pulmonar, con diagnóstico clínico de comunicación interauricular. El ecocardiograma mostró estenosis de venas pulmonares izquierdas. El cateterismo cardiaco detectó fístulas arteriovenosas en la región apical del pulmón derecho. La imagen de resonancia magnética y la angiografía mostraron un vaso arterial aberrante paralelo a la aorta abdominal y con flujo dirigido al lóbulo pulmonar derecho. La angiotomografía reportó confluencia de las venas pulmonares del lado derecho. Se realizó lobectomía derecha. La paciente falleció en el posoperatorio debido a una hemorragia masiva pulmonar. Esta paciente es la primera descrita en la literatura con estenosis de venas pulmonares congénita asociada a secuestro pulmonar. La ecocardiografía es el estudio diagnóstico ideal inicial en los pacientes con estenosis congénita de venas pulmonares.
\end{abstract}

Palabras clave: Estenosis de venas pulmonares. Cardiopatía congénita. Enfermedad cardiaca pulmonar. Secuestro pulmonar.

\begin{abstract}
The objective is demonstrate the diagnostic process and evolution of a patient with a diagnosis of congenital pulmonary vein stenosis and broncho-pulmonary vascular malformation. One year old female patient with repeated bronchopneumonia, acrocyanosis, split S2, cardiomegaly, pulmonary hypertension, with a clinical diagnosis of atrial septal defect. The echocardiogram demonstrated left sided vein pulmonary stenosis. The cardiac catheterization demonstrated arterial-venous fistulas apical on the right lung. Magnetic Resonance image and angiography showed an aberrant arterial vessel parallel to the abdominal aorta which flow the right pulmonary lobe. The cardiac tomography angiography reported confluence of right-sided pulmonary veins. A lobectomy is performed. Patient died in post-operative due to massive pulmonary hemorrhaging. This is the first patient mentioned in written literature with pulmonary vein stenosis associated with pulmonary sequestration, with normal venous connection. Echocardiography represents the specific standard study ideal for initial diagnostic for patients with pulmonary vein stenosis.
\end{abstract}

Key words: Pulmonary vein stenosis. Congenital heart disease. Pulmonary heart disease. Pulmonary sequestration.

\section{Correspondencia:}

*Jesús De Rubens-Figueroa

E-mail: derubens @ hotmail.com
Disponible en internet: 16-03-2021

\section{Arch Cardiol Mex. 2021;91(2):355-360
www.archivoscardiologia.com \\ Arch Cardiol Mex. 2021;91(2):355-360
www.archivoscardiologia.com}

Fecha de recepción: 28-07-2020

Fecha de aceptación: 25-01-2021 DOI: $10.24875 /$ ACM.20000362 


\section{Introducción}

La estenosis de venas pulmonares tiene una incidencia poblacional del $0.03 \%{ }^{1}$, y puede ser congénita o adquirida (secundaria a procedimientos con ablación por radiofrecuencia, cirugía de las venas pulmonares anómalas y estados posquirúrgicos de Fontan, Mustard o Senning). Pueden estar involucradas una o varias venas pulmonares, y presentarse aislada o asociada a una cardiopatía ${ }^{2-5}$.

La estenosis congénita de venas pulmonares (ECVP) es una enfermedad cardiaca rara, difícil de diagnosticar y con frecuencia con alta mortalidad. Lo esperado es encontrar la estenosis entre la unión de la vena y el atrio izquierdo, o su asociación con hipoplasia difusa de las venas pulmonares, generalmente asociada con algunas lesiones cardiacas ${ }^{6}$.

La primera publicación se realizó en 1951 sobre un niño de 8 años que falleció como consecuencia de las complicaciones de la enfermedad?.

Hasta donde sabemos no existe otra publicación de algún paciente con ECVP sin conexión anómala de venas pulmonares y con secuestro pulmonar, por lo que presentamos nuestra experiencia de una paciente con esta patología.

\section{Caso clínico}

Niña nacida a las 30 semanas de edad gestacional con un peso de $1400 \mathrm{~g}$, cianosis al llanto desde el nacimiento, falla a la alimentación y que ha presentado bronconeumonías de repetición.

Al año de edad, la exploración física revela peso de $6900 \mathrm{~g}$, talla de $77 \mathrm{~cm}$, frecuencia cardiaca de $148 \mathrm{la}-$ tidos por minuto, frecuencia respiratoria de 70 respiraciones por minuto y presión arterial de $87 / 53 \mathrm{mmHg}$. Presenta acrocianosis al llanto. La saturación de oxígeno es del $83 \%$ al aire ambiente y del $85 \%$ con oxígeno. Aleteo nasal, deformidad torácica anteroposterior, sin soplos, $2 \mathrm{R}$ metálico, pulsos presentes y normales.

La radiografía de tórax mostró cardiomegalia con índice cardiotorácico de 0.64 e hilio pulmonar congestivo y líneas B de Kerley.

El electrocardiograma mostró una frecuencia cardiaca de 140 latidos por minuto, eje de A QRS- $150^{\circ}$, segmento S-T negativo en derivaciones derechas y crecimiento de cavidades derechas.

En ese momento se diagnosticó comunicación interauricular e hipertensión arterial pulmonar y a descartar conexión anómala de venas pulmonares. El tratamiento inicial consistió en captopril, furosemida y espironolactona.

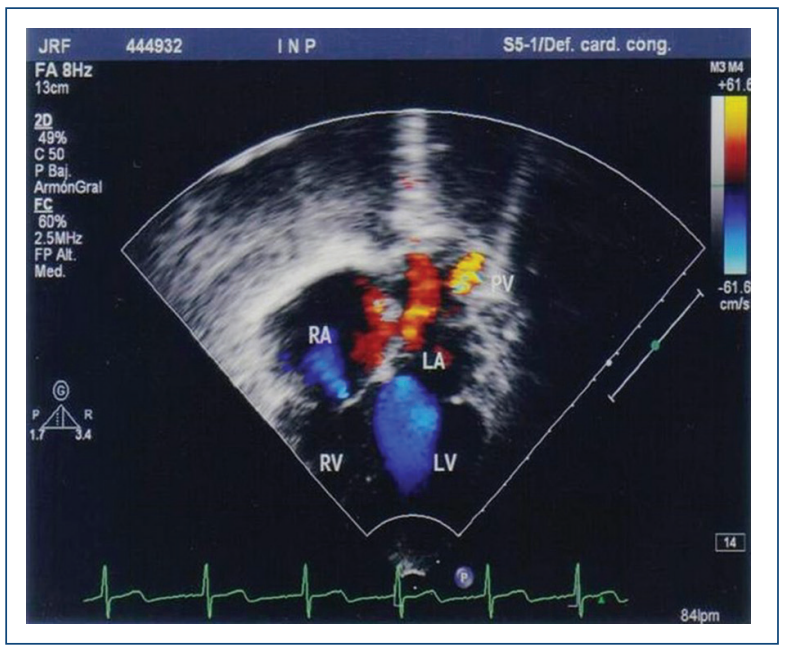

Figura 1. Ecocardiograma en color bidimensional, en el cual se observa una turbulencia en color en la vena pulmonar izquierda y crecimiento de las cavidades derechas. LA: atrio izquierdo; LV: ventrículo izquierdo; RA: atrio derecho; RV: ventrículo derecho; PV: vena pulmonar.

El ecocardiograma mostró una comunicación interauricular de $10 \mathrm{~mm}$, tipo seno venoso con cortocircuito mixto, hipertensión arterial pulmonar de $68 \mathrm{mmHg}$ y estenosis de venas pulmonares izquierdas (por presentación la más frecuente) ${ }^{5}$ demostrada por eco-color con turbulencia y $25 \mathrm{mmHg}$ de gradiente máximo (Fig. 1).

El cateterismo cardiaco (que es el estudio referente) ${ }^{5}$ demostró la comunicación interauricular, las cuatro venas pulmonares conectadas al atrio izquierdo, estenosis de venas pulmonares izquierdas y fístulas pulmonares derechas, con presión sanguínea de 100/46 $\mathrm{mmHg}$ con media de $64 \mathrm{mmHg}$ (Fig. 2). La resistencia pulmonar fue de 7 UW y la sistémica de 9.1 UW.

Tanto la angiografía de grandes vasos como la resonancia magnética mostraron la presencia de arteria aberrante con origen en la aorta abdominal, que irriga la porción media del lóbulo inferior derecho; se consideró secuestro pulmonar.

El ultrasonido abdominal mostró un vaso aberrante con origen en la aorta abdominal por arriba del tronco celíaco, y fístulas arteriovenosas confirmadas.

La tomografía computarizada mostró estenosis de las dos venas pulmonares izquierdas conectadas a la aurícula izquierda, ambas son confluentes de un solo tronco con estenosis de $1.9 \mathrm{~mm}$ de diámetro, formación de fistulas arteriovenosas originadas de un vaso arterial, ramificaciones de la aorta abdominal que cruzan el diafragma al lado derecho de la base pulmonar y se 


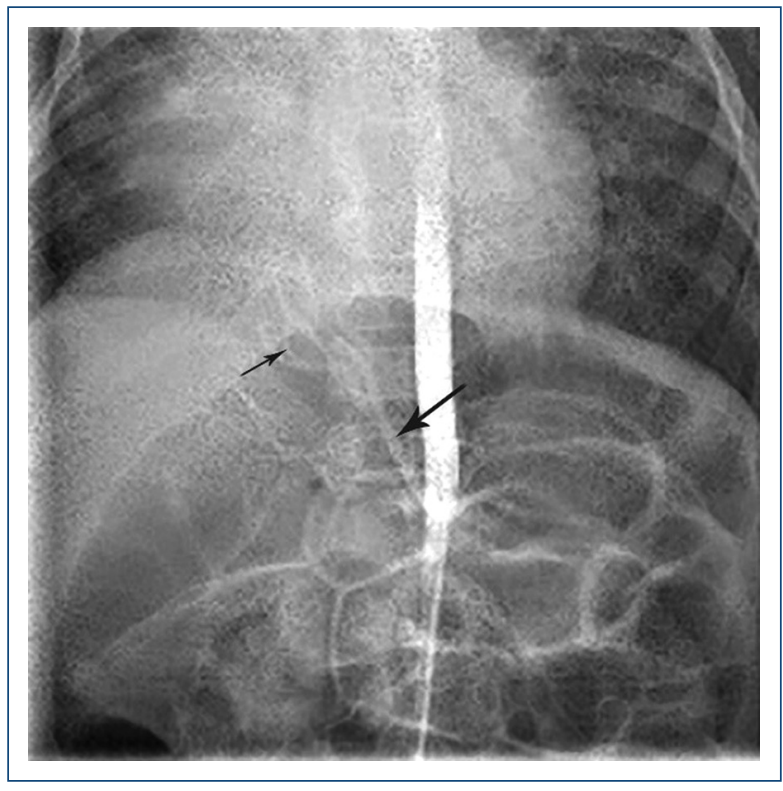

Figura 2. En el cateterismo cardiaco se observa una fístula arterial derecha (flecha gruesa) que emerge de la aorta abdominal hacia la base pulmonar derecha (secuestro) (flecha fina).

divide en dos pequeñas ramas que se anastomosan al vaso venoso (Fig. 3).

Debido a que se decidió realizar una neumonectomía por el secuestro pulmonar (se descartó la posibilidad de colocar un oclusor vascular por cateterismo intervencionista para disminuir el flujo del secuestro pulmonar), se llevó a cabo una toracotomía posterolateral derecha y se encontró que el lóbulo inferior derecho estaba adherido a la pleura mediastinal; se realizó neumonectomía derecha, ampliación de la estenosis venosa pulmonar con ligadura y corte del vaso anómalo.

Ingresó a terapia intensiva, donde presentó hemorragia pleural $(170 \mathrm{ml})$, acidosis respiratoria e hipoglucemia, sangrado a través de la cánula endotraqueal y derrame pleural de pulmón izquierdo. La paciente presentó insuficiencia respiratoria durante 8 minutos con bradicardia grave, sin respuesta al tratamiento.

La autopsia reveló múltiples malformaciones congénitas: estenosis de venas pulmonares izquierdas y malformación vascular arteriovenosa en la base del pulmón derecho, las cuales fueron corregidas quirúrgicamente. Se informó comunicación interauricular de $10 \mathrm{~mm}$, hipertensión arterial pulmonar GI-II secundaria a enfermedad vascular pulmonar, hemosiderosis pulmonar e hipertrofia de cavidades derechas.

La causa de la muerte fue una hemorragia masiva pulmonar y mediastinal posoperatoria, hemotórax



Figura 3. Tomografía computarizada en la que se observan la dilatación de las venas pulmonares izquierdas (flecha gruesa), que son confluentes, y el vaso aberrante (flecha fina).

derecho, edema pulmonar y cerebral con degeneración hipóxico-neuronal.

\section{Discusión}

La ECVP es un problema alrededor de los días 28 a 30 de edad gestacional, en el momento en que ocurre la separación embrionaria de las venas pulmonares ${ }^{8}$. Las manifestaciones clínicas de esta patología son soplo diastólico o continuo, insuficiencia cardiaca derecha, bajo peso, dificultad respiratoria, neumonía crónica, hemoptisis, taquipnea, hipertensión y edema pulmonar ${ }^{1,2,9-11}$.

El diagnóstico se estableció con ecocardiografía Doppler-color, que mostró la ECVP y una importante obstrucción por arriba de $16 \mathrm{mmHg}$.

La velocidad del flujo no debe ser superior a $1.5 \mathrm{~m} / \mathrm{s}$ y estudios previos han mostrado, en los ecocardiogramas realizados, una progresión significativa de la obstrucción, hipertrofia del ventrículo derecho y aumento de la presión sistólica de la arteria pulmonar ${ }^{2,3}$.

La ecocardiografía es un estudio específico estándar para el diagnóstico inicial de esta patología, pero con ciertas limitaciones: localización del flujo y alineación 


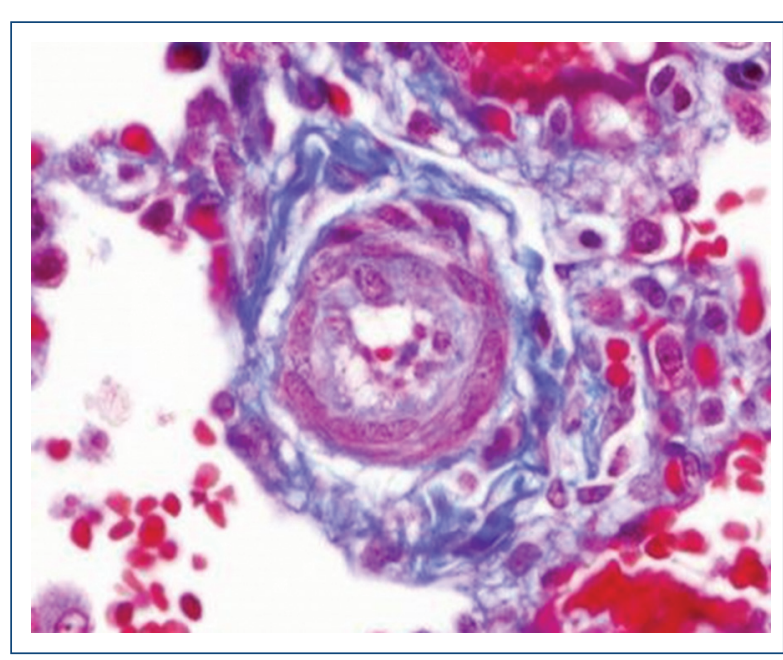

Figura 4. El estudio patológico muestra inflamación granulomatosa y proliferación focal fibrosa de la íntima (Dra. De León-Bojorge, Dpto. Patología).

con el Doppler a $20^{\circ}$ o menos (en pacientes con atresia pulmonar, el flujo sanguíneo podría ser menor) ${ }^{3,10}$.

El cateterismo es el método de referencia para las características de la estenosis, y el intervencionismo es útil en manos expertas, pero se considera una medida paliativa para el trasplante cardiopulmonar ${ }^{5}$.

La tomografía computarizada con reconstrucción tridimensional es la técnica de imagen complementaria de las estructuras de la anatomía del sistema vascular, así como de las venas pulmonares ${ }^{5}$. Si entendemos que es necesario disponer de los datos de la posición de las venas pulmonares con respecto al atrio para determinar el óptimo abordaje quirúrgico, las técnicas de imagen (ecocardiografía transesofágica, tomografía computarizada, resonancia magnética e imagen perfusoria) son esenciales para alcanzar la fineza en el diagnóstico final ${ }^{12,13}$.

La ECVP es un proceso patológico de engrosamiento muscular, proliferación-tortuosidad miofibroblástica de la neoíntima y alteración en la matríz extracelular, aumento de factor plaquetario, de factor vascular endotelial y de fibroblastos, con activación de receptores de la tirosina cinasa e hipertensión arterial pulmonar ${ }^{14,15}$.

El estudio patológico mostró inflamación granulomatosa y proliferación focal fibrosa de la íntima, la cual se describe como una enfermedad intraparenquimatosa ${ }^{2,4,8}$ (Fig. 4). El mecanismo de desarrollo no es bien entendido. Aparentemente, la ECVP es una lesión progresiva ${ }^{9,10,16}$.

La ECVP se asocia con frecuencia a defectos cardiacos, como comunicaciones atriales o ventriculares, persistencia del conducto arterioso y ventrículos únicos
$(30-80 \%)^{1,3,4,6,7,17}$. La mayoría son de afección unilateral $^{18,19}$. Nosotros no encontramos casos asociados con secuestro pulmonar.

La gravedad del caso dependerá del número de venas pulmonares afectadas, la magnitud de la obstrucción $\left(30-46 \mathrm{~mm} / \mathrm{m}^{2}\right)$ y el tipo de lesión (tubular, constricción venosa arterial 0 lesiones múltiples hipoplásicas) 2,20,21.

Los predictores para reintervención o incluso de mal pronóstico son diagnóstico tardío, antecedente de prematurez con distrés respiratorio, edad menor de 6 meses, peso inferior a $3 \mathrm{~kg}$, hipertensión pulmonar, afección en la presión sistólica ventricular derecha o afección bilateral de las venas pulmonares, y cardiopatía congénita con cortocircuito de izquierda a derecha 2 ,11,14,19,22,23.

Nuestra paciente cumplía varios factores de mal pronóstico: presencia de secuestro pulmonar asociado a hipertensión pulmonar, comunicación interauricular, antecedente de prematurez con peso bajo y diagnóstico al año de edad.

Los pacientes con estenosis o hipoplasia de venas pulmonares son de difícil manejo $0^{2,4,6,24,25}$. Algunas opciones se basan en el uso de betabloqueadores, digoxina, anticoagulantes, antiplaquetarios ${ }^{12}$, quimioterapia, oxígeno suplementario, bosentán para el manejo de la hipertensión pulmonar ${ }^{5}$, angioplastia con balón o colocación de stents (un desafío en niños) con sirolimus (rapamicina) para prevenir la estenosis (no son recomendables cuando las estenosis se localizan en los ostia venosos) ${ }^{18,26}$. Algunos autores consideran mejor la cirugía que el cateterismo por tener experiencias previas de reestenosis con el cateterismo ${ }^{16}$. En todo el mundo la experiencia es limitada para la intervención percutánea en niños ${ }^{12}$. Se requiere experiencia para las diferentes técnicas quirúrgicas: endovenectomía o venoplastia con utilización de pericardio o parches de safena, o marsupialización con pericardio, reparación con parche (técnica clásica) o sin sutura pericárdica (técnica actual) 1,4,6,12,25-28. Nosotros todavía carecemos de experiencia para considerar algunas de estas técnicas. Muchos de estos procedimientos son paliativos hasta el tratamiento final de trasplante de corazón-pulmón ${ }^{16}$.

En todos estos pacientes hay una incidencia alta de reestenosis $(50 \%)$, debido a la gran proliferación de la neoíntima, 4 a 8 semanas después del tratamiento ${ }^{1,2,8,9,17,28}$, lo cual presta la posibilidad a una segunda intervención antes de 6 meses, con reoperación del $40 \%$ y mortalidad del $30 \%{ }^{29}$. Esto hace que el tratamiento de esta patología sea difícil y tenga un mal pronóstico ${ }^{8,9,21}$. 
Se ha publicado una escala para el pronóstico posquirúrgico en cuanto a riesgo de reestenosis y reoperación que toma en cuenta el gradiente de obstrucción de las venas pulmonares, si la enfermedad es unilateral o bilateral, el aspecto de la lesión difuso o focal (lo más grave es atresia u oclusión) y si la técnica quirúrgica se lleva a cabo con o sin sutura ${ }^{29}$.

En cuanto al pronóstico, se ha reportado que en los prematuros la sobrevida a 2 años es del $43 \%$, en los niños menores de 1 año la mortalidad es de alrededor del $65 \%$ y en los mayores de esa edad es del $17 \%{ }^{16}$.

La mortalidad global a 7 meses del diagnóstico por insuficiencia cardiaca es del $44 \%{ }^{5}$ y a 3 años es del $49 \%{ }^{23}$. La sobrevida a 5 años en los grupos de Boston y Toronto fue del $58 \%$ y el $64 \%$, respectivamente ${ }^{22}$. Con broncodisplasia pulmonar e hipertensión arterial pulmonar la cirugía es muy riesgosa y quizá se requiere cateterismo intervencionista previo ${ }^{16}$. Nuestra experiencia en una serie de pacientes es muy limitada.

Desde la primera cirugía correctiva de ECVP en 1971 hasta la fecha el pronóstico sigue siendo pobre.

La neumonectomía está indicada en casos con hemoptisis. El trasplante es otra opción, pero con las posibles complicaciones conocidas, como inmunosupresión, neoplasias y bronquitis obliterativa ${ }^{1,24}$.

En nuestra paciente, con diagnóstico de comunicación interauricular, había una sospecha clínica de obstrucción del lado izquierdo del corazón debido a los datos de hipertensión venocapilar pulmonar observados en la radiografía de tórax. El ecocardiograma apoyó el diagnóstico inicial.

Nosotros consideramos apropiada la corrección quirúrgica de la ECVP al mismo tiempo que llevar a cabo la neumonectomía, aunque desafortunadamente la paciente falleció debido a una hemorragia en el sitio de la incisión durante el posoperatorio inmediato.

\section{Conclusiones}

La estenosis de venas pulmonares debe ser descartada en todo niño prematuro con hipertensión pulmonar con o sin cardiopatía congénita, y debe realizarse un ecocardiograma en manos expertas para confirmar el diagnóstico.

Siempre, ante la sospecha de hipertensión venocapilar pulmonar, hay que revisar con ecocardiografía color y Doppler la luz de las venas pulmonares.

El tratamiento de la estenosis de venas pulmonares es uno de los mayores desafíos entre las enfermedades cardiovasculares. Se requiere experiencia interinstitucional.

\section{Financiamiento}

Ninguno.

\section{Conflicto de intereses}

Declaramos que no existe ningún conflicto de intereses.

\section{Responsabilidades éticas}

Protección de personas y animales. Los autores declaran que para esta investigación no se han realizado experimentos en seres humanos ni en animales.

Confidencialidad de los datos. Los autores declaran que han seguido los protocolos de su centro de trabajo sobre la publicación de datos de pacientes.

Derecho a la privacidad y consentimiento informado. Los autores declaran que en este artículo no aparecen datos de pacientes.

\section{Bibliografía}

1. Holt $\mathrm{BD}$, Moller $\mathrm{HJ}$, Larson $\mathrm{S}$, Johnson $\mathrm{CM}$. Primary pulmonary vein stenosis. Am J Cardiol. 2007;99:568-72.

2. Latson AL, Prieto LR. Congenital and acquired pulmonary vein stenosis. Circulation. 2007;115:103-8.

3. Vick III WG, Murphy JD, Ludomirsky A, Morrow WR, Morriss JM, Danford $A D$, et al. Pulmonary venous systemic ventricular inflow obstruction in patients with congenital heart disease: detection by combined two-dimensional and Doppler echocardiography. J Am Coll Cardiol. 1987;9:580-7.

4. Van Son, AJ, Danielson KG, Puga JF, Edwards DW, Driscoll JD. Repair of congenital and acquired pulmonary vein stenosis. Ann Thorac Surg. 1995;60:144-50

5. Laux D, Rocchisani MA, Boudjemline Y, Gouton M, Bonnet D, Ovaert C. Pulmonary hypertension in the preterm infant with chronic lung disease can be caused by pulmonary bien stenosis: a must-know entity. Pediatr Cardiol. 2016;37:313-21.

6. Driscoll JD, Hesslein SP, Mullins E CH. Congenital stenosis of individual pulmonary veins: clinical spectrum and unsuccessful treatment by transvenous balloon dilation. Am J Cardiol. 1982;49:1767-72.

7. Reye D. Congenital stenosis of the pulmonary veins in their extrapulmonary course. Med J Aust. 1951;1:801-2.

8. Sadr MI, Tan EP, Kieran WM, Jenkins JK. Mechanism of pulmonary vein stenosis in infants with normally connected veins. Am J Cardiol. 2000;86:577-9.

9. Bini MR, Cleveland CD, Ceballos R, Bargeron ML, Pacifico DA, Kirklin WJ. Congenital pulmonary vein stenosis. Am J Cardiol. 1984;54:369-75.

10. Smallhorn FJ, Pauperio H, Benson L, Freedom MR, Rowe DR. Pulsed Doppler assessment of pulmonary vein obstruction. Am Heart J. 1985;110:483-6.

11. Alsoufi B. Cracking the mystery of pulmonary vein stenosis. Semin Thorac Cardiovasc Surg. 2019;31:274-6.

12. Pazos LP, García RC, Guitián G, Paredes GE, Álvarez MA, Rodríguez AM, et al. Pulmonary vein stenosis: etiology, diagnosis and management. World J Cardiol. 2016;8:81-8.

13. Hong H. Commentary: Management outcomes of primary pulmonary vein stenosis: what we know now and what we can expect in the future. J Thorac Cardiovasc Surg. 2020;159:1039.

14. Tsang TV, Tran KP. Pulmonary vein stenosis: challenges ahead. J Thorac Cardiovasc Surg. 2015;150:776.

15. Pogoriler JE, Kulik TJ, Casey AM, Baird CW, Mullen MP, Jenkins KJ, et al. Lung pathology in pediatric pulmonary vein stenosis. Pediatr Dev Pathol 2016;19:219-29.

16. Bonello B, Trivedi RK, Fraisse A. Multiple and aggressive pulmonary vein transcatheter interventions as bridge to transplantation in primary diffuse pulmonary vein stenosis. Cathet Cardiovasc Interv. 2015;86:e190-3.

17. Park CS, Neches HW, Lenox CC, Zuberbuhler RJ, Siewers DR, Bahnson TH. Diagnosis and surgical treatment of bilateral pulmonary vein stenosis. J Thorac Cardiovasc Surg. 1974;67:755-61. 
Arch Cardiol Mex. 2021;91(3)

18. Callahan R, Esch J, Wang G, Ireland $\mathrm{MCH}$, Gauvreau $\mathrm{K}$, Jenkins $\mathrm{K}$. Systemic sirolimus to prevent in stent stenosis in pediatric pulmonary vein stenosis. Pediatr Cardiol. 2020;41:282-9.

19. Rosenblum MJ, Altin FHK, Gillespie S, Heaton BH, Kanter A, Sinha R, et al. Management outcomes of primary pulmonary vein stenosis. $J$ Thorac Cardiovasc Surg. 2020;159:1029-36.

20. Breinholt PJ, Hawkins AJ, Minich LA, Tani LY, Orsmond SG, Ritter S et al. Pulmonary vein stenosis with normal connection: associate cardiac abnormalities and variable outcome. Ann Thorac Surg. 1999;68:164-8.

21. Seale AN, Daubeney PEF, Magee AG, Rigby ML. Pulmonary vein stenosis: initial experience with cutting balloon angioplasty. Heart. 2006;92:815-20.

22. De Campli MW. Pulmonary vein stenosis: plea for a multi-institutiona registry. J Thorac Cardiovasc Surg. 2016;151:667-8.

23. Seale AN, Webber SA, Uemura H, Partridge J, Roughton M, Ho SY. Pulmonary vein stenosis: the UK, Ireland and Sweden collaborative study. Heart. 2009;95:1944-9.
24. Mendeloff NE, Spray LTH, Huddleston BCH, Bridges DN, Canter BCH, Mallory BG. Lung transplantation for congenital pulmonary vein stenosis. Ann Thorac Surg. 1995;60:903-7.

25. Devaney JE, Chang CA, Ohye GR, Bove LE. Management of congenital and acquired pulmonary vein stenosis. Ann Thorac Surg. 2006; 81:992-6.

26. Ungerleider MR, Johnston AT, O'Laughlin PM, Jaggers JJ, Gaskin RP. Intraoperative stents to rehabilitate severely stenotic pulmonary vessels. Ann Thorac Surg. 2001;71:476-81.

27. Kirk RK, Kirshbom MP, Kogon EB. Surgical repair of pulmonary venous stenosis: a word of caution. Ann Thorac Surg. 2014;98:1687-91.

28. Victor $S$, Nayak MV. Deringing procedure for congenital pulmonary vein stenosis. Tex Heart Inst J. 1995;22:166-9.

29. Kalfa D, Belli E, Bacha E, Lambert V, Duccio di C, Kostolny M, et al. Primary pulmonary vein stenosis: outcomes, risk factors and severity score in a multicentric study. Ann Thorac Surg. 2017;104:182-9. 